\title{
Carbon-ion radiotherapy in osteosarcoma of the mandible: a case report
}

\author{
Tae-Wook Ha, Slmaro Park, Min Yeong Youn, Dong Wook Kim, Hyung Jun Kim \\ Department of Oral \& Maxillofacial Surgery, College of Dentistry, Yonsei University, Seoul, Korea
}

\begin{abstract}
J Korean Assoc Oral Maxillofac Surg 2021;47:315-320)
Carbon-ion radiotherapy (CIRT) is on the rise as a treatment choice for malignant tumor. Compared to conventional radiotherapy, particle beams have different physical and biological properties. Particle beam provides a low entry dose, deposits most of the energy at the endpoint of the flight path, and forms an asymptotic dose peak (the "Bragg peak"). Compared to protons, carbon with its larger mass decreases beam scattering, resulting in a sharper dose distribution border. We report a 50-year-old male who underwent CIRT without surgical resection on osteosarcoma of the mandible. After CIRT, the patient's pain was gone, and the malignant mass remained stable with accompanying necrosis. Nine months later, however, magnetic resonance imaging demonstrated progression of the left mandibular osteosarcoma with pulmonary metastases. After multidisciplinary discussion, concurrent chemoradiotherapy was conducted. While necrotic bone segments came out of the mandible during subsequent periodic outpatient visits, the tumor itself was stable. Thirty months after his first visit and diagnosis, the patient is waiting for chemotherapy. Although CIRT is superior in treating radioresistant hypoxic disease, CIRT is in its infancy, so care must be taken for its indications and complications.
\end{abstract}

Key words: Osteosarcoma, Heavy ion radiotherapy, Radiotherapy, Head and neck neoplasms

[paper submitted 2019. 7. 17 / revised 2019. 9. 11 / accepted 2019. 10. 15]

\section{Introduction}

Osteosarcoma is a rare malignant bone tumor that most commonly arises in the extremities and rarely in the head and neck region ${ }^{1}$. Osteosarcoma of the head and neck region comprises less than $10 \%$ of all osteosarcomas and $1 \%$ of all head and neck malignancies ${ }^{2}$.

The two main therapies for head and neck malignancies are surgery and radiation. Since it is known that osteosarcoma is a relatively radio-resistant malignant tumor, radiotherapy is usually not considered as the first choice of treatment ${ }^{3}$. In a retrospective study of 26 Korean patients with osteogenic sarcoma of the jaw, Jeong et al. ${ }^{4}$ found that the most important factor for disease survival was surgical resection with a clear margin. Carbon-ion radiotherapy (CIRT) has recently emerged as an alternative therapy due to its concentrated dis-

\section{Hyung Jun Kim}

Department of Oral \& Maxillofacial Surgery, College of Dentistry, Yonsei University, 50-1 Yonsei-ro, Seodaemun-gu, Seoul 03722, Korea

TEL: +82-2-2228-3132

E-mail:kimoms@yuhs.ac

ORCID: https://orcid.org/0000-0002-3364-9995

(c) This is an open-access article distributed under the terms of the Creative Commons Attribution Non-Commercial License (http://creativecommons.org/ licenses/by-nc/4.0/), which permits unrestricted non-commercial use, distribution, and reproduction in any medium, provided the original work is properly cited.

Copyright (C) 2021 The Korean Association of Oral and Maxillofacial Surgeons. tribution and strong biological effects. Photon radiotherapy with a dose of 50-60 Gy (gray) is currently considered to have a minor role in multi-disciplinary approaches ${ }^{5,6}$.

Here, we report a 50-year-old male who underwent CIRT without resective surgery on an osteosarcoma.

\section{Case Report}

A 50-year-old male was referred for evaluation and treatment of a malignant mesenchymal tumor of the left mandible, which was diagnosed after a peroral incisional biopsy that suggested sarcoma in November 2016. He reported symptoms such as facial swelling, weakness of ipsilateral facial nerve (temporal branch) and severe pain (visual analogue scale [VAS], 7-8). Magnetic resonance imaging (MRI) revealed an osteolytic mass centered at the left mandibular condyle with involvement of the infratemporal fossa and pterygoid muscles.(Fig. 1. A) It was in close proximity with the inferior alveolar nerve, left parotid gland, pterygopalatine fossa, and skull base (cT1N0M0, high grade, stage IIA). While we originally recommended that he undergo surgical resection with microvascular reconstruction, the patient declined and underwent definitive CIRT at an institution in Japan. A total dose of $70.4 \mathrm{GyE}$ (gray equivalents) in 16 frac- 

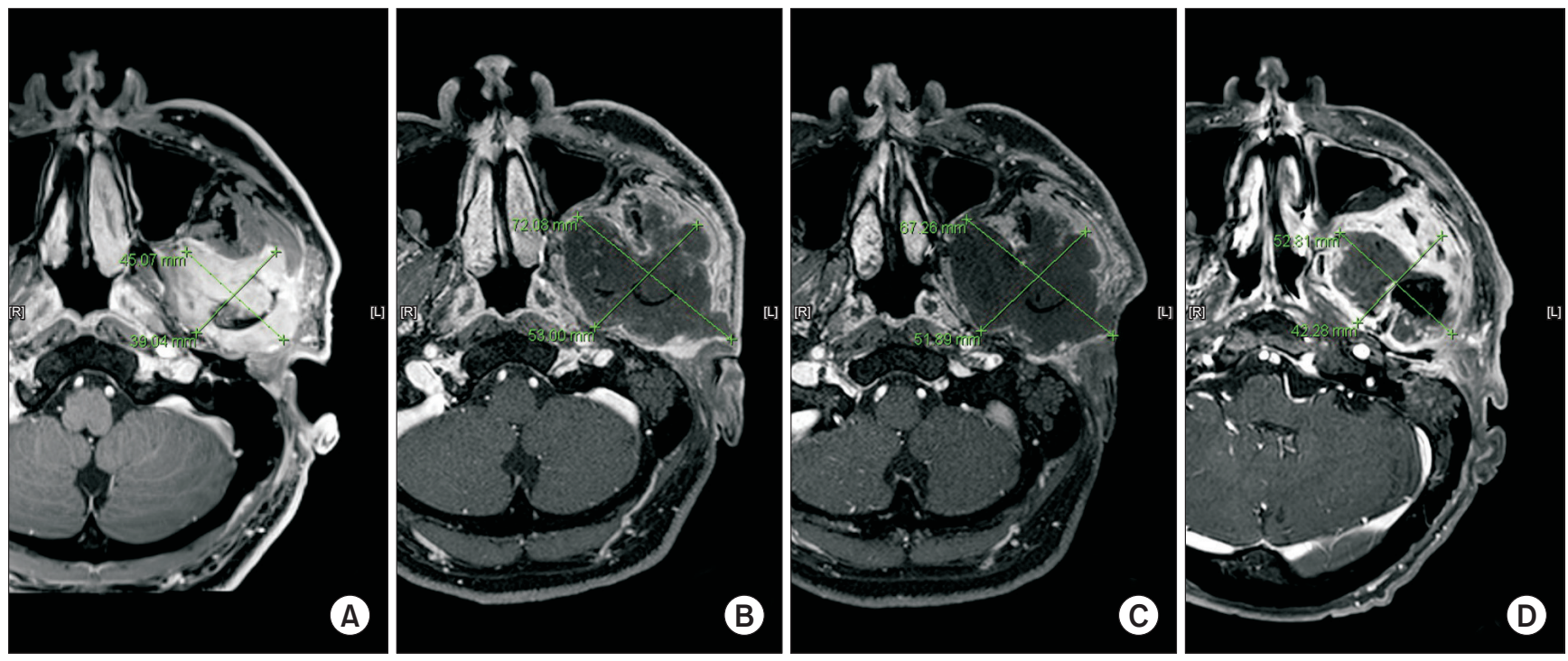

Fig. 1. Measured tumor size in magnetic resonance imaging. A. October 2016, before carbon-ion radiotherapy $(\mathrm{CIRT}) .4 .5 \mathrm{~cm} \times 3.9 \mathrm{~cm}$. B. April 2017, 3 months after CIRT. $7.2 \mathrm{~cm} \times 5.3 \mathrm{~cm}$. C. October 2017, 9 months after CIRT. No change in overall size of tumor. D. October 2018, 21 months after CIRT. Suspected recurrence of tumor.

Tae-Wook Ha et al: Carbon-ion radiotherapy in osteosarcoma of the mandible: a case report. J Korean Assoc Oral Maxillofac Surg 2021
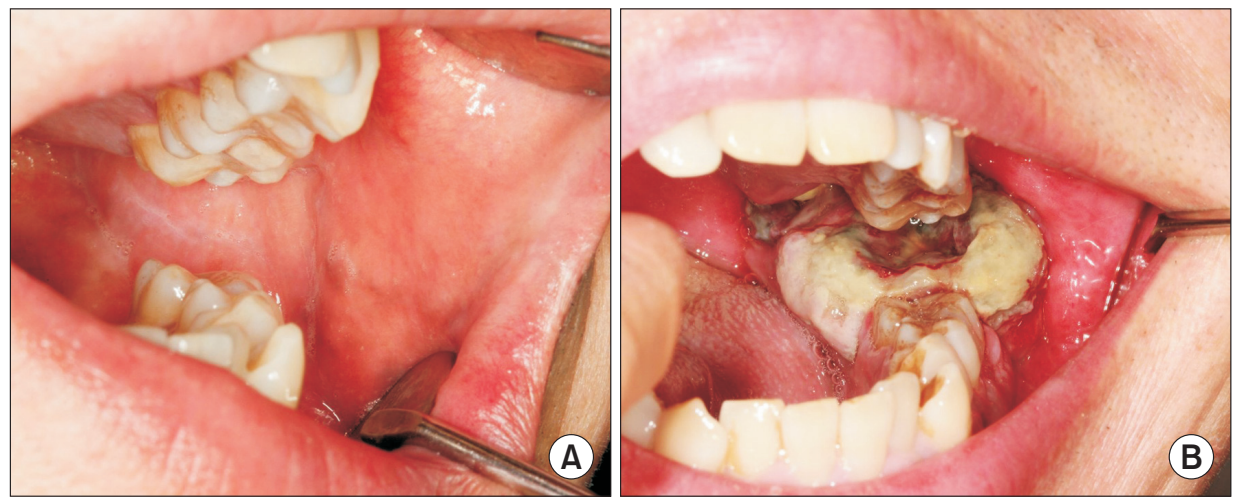

Fig. 2. A. Apirl 2017. The patient had newly-developed symptoms such as dysesthesia on left buccal cheek, limited mouth opening, xerostomia, oral ulcers and ipsilateral otitis externa with hearing loss. B. January 2018. Peroral biopsy of ulcerative soft tissue mass was done. Tae-Wook Ha et al: Carbon-ion radiotherapy in osteosarcoma of the mandible: a case report. J Korean Assoc Oral Maxillofac Surg 2021 tions was administered over 1 month (from December 16, 2016 through January 13, 2017).

In February 2017, he re-visited our institution for a checkup and revealed new symptoms such as dysesthesia on the left buccal cheek, limited mouth opening, xerostomia, oral ulcers and ipsilateral otitis externa with hearing loss, which were considered to be CIRT side effects.(Fig. 2. A) While showing these new symptoms, the patient insisted on a reduced pain score of VAS 2-3, which had been 7-8 before treatment.

In April 2017, an MRI was taken and compared with one taken 6 months previously. The malignant mass increased in size $(4.5 \mathrm{~cm} \times 3.9 \mathrm{~cm}$ to $7.2 \mathrm{~cm} \times 5.3 \mathrm{~cm})$, with central necrosis. Furthermore, involvement of the infratemporal fossa, pterygoid process, parotid gland and abutting into the posterior wall of the left maxillary sinus was observed.(Fig. 1. B) No signs of lymph node metastasis were evident. The patient claimed no pain.

In October 2017, an MRI for periodic follow-up showed no change in the overall size of the large expansile necrotic tumor.(Fig. 1. C) However, in a positron emission tomographycomputed tomography (PET-CT) whole body scan, newly developed ${ }^{18} \mathrm{~F}$-fluorodeoxyglucose (FDG) uptake were found in the ipsilateral mandibular ramus and right lower lobe of the lung, suspicious for recurrence and metastasis, respectively.

A multidisciplinary discussion transpired between the Departments of Oral and Maxillofacial Surgery, Medical Oncology, Radiation Oncology, and Thoracic and Cardiovascular Surgery. The patient was able to tolerate mouth opening for a peroral biopsy to determine the subtype, which turned out to be osteosarcoma, epithelioid type.(Fig. 2. B) The first palliative chemotherapy, with cisplatin and doxorubicin, did not seem to have an effect on the lesion. Palliative radiotherapy 

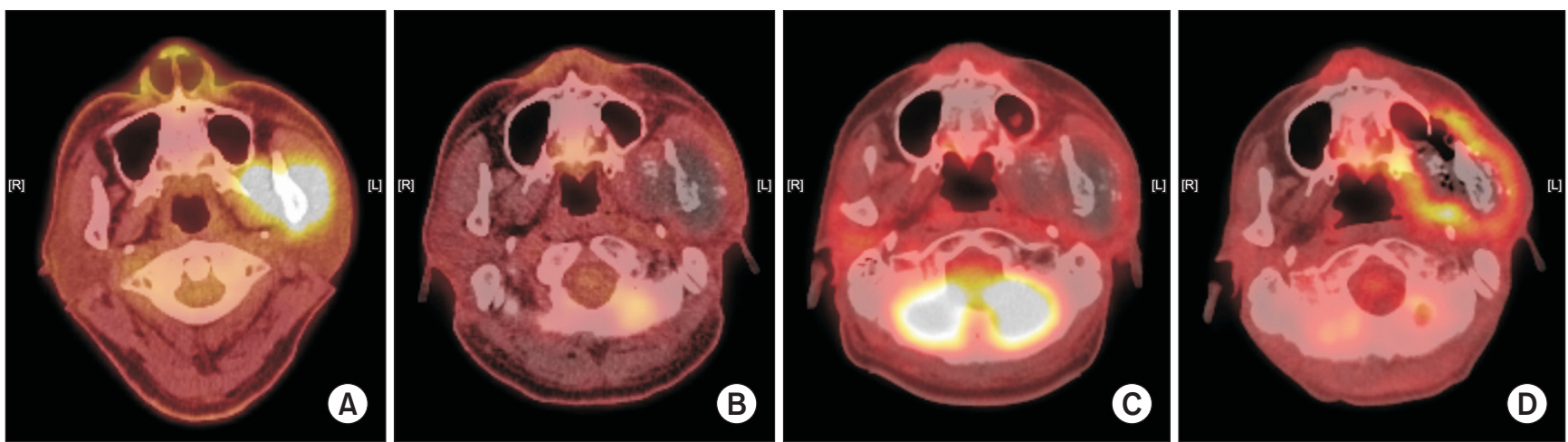

Fig. 3. A. November 2016. Mass with increased ${ }^{18} \mathrm{~F}$-fluorodeoxyglucose (FDG) uptake in the left masticator space, suggesting malignancy. No sign of lymph node or distant metastasis was observed. B. October 2017, 9 months after carbon-ion radiotherapy (CIRT). C. February 2018, 13 months after CIRT. D. October 2018, 21 months after CIRT. Recurrent tumor with metastatic lymphadenopathy was suspected. Tae-Wook Ha et al: Carbon-ion radiotherapy in osteosarcoma of the mandible: a case report. J Korean Assoc Oral Maxillofac Surg 2021

of total 2,500 cGy over 8 days (February 28 to March 7, 2018) was then conducted. After the eighth palliative chemotherapy, a PET-CT taken in March 2018 demonstrated slight decreases in the sizes of the left mandibular mass and lung nodules.

A second multidisciplinary meeting was held in September 2018 to discuss treatment planning, including the need for surgical resection of the primary lesion and lung nodules. The plan was to perform lung metastasectomy with resection of the primary lesion if possible, but the patient refused to undergo surgery. On MRI and PET-CT taken in October, 1 month after the multidisciplinary meeting, recurrent tumor with metastatic lymphadenopathy was suspected.(Fig. 1. D, 3. D) In December 2018, necrotic change of the tumor was observed, and the patient received intravenous antibiotic therapy under admission. During outpatient visits from December 2018 to April 2019, necrotic bone falling out of the mandible was observed.

On chest CT and neck CT taken in January 2019, the overall disease was stable, implying successful local control of the primary lesion as well as the lung metastases, for the time being. The patient refused to undergo surgical treatment and is now awaiting palliative chemotherapy when his general condition improves.

\section{Discussion}

According to the National Comprehensive Cancer Network (NCCN) guidelines, both primary and adjuvant treatments can be altered according to the grade and subtype of osteosarcoma. The epithelioid type is categorized as a rare variant of the central (intramedullary) type with a high histological

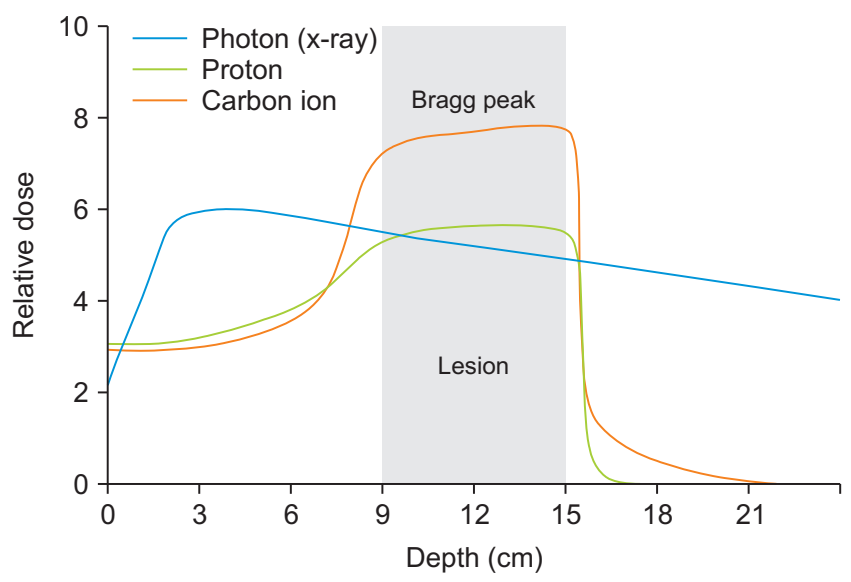

Fig. 4. A comparison of the physics of X-ray and particle beam doses. The proton and carbon ion beams have a significantly lower entrance dose, and no exit dose.

Tae-Wook Ha et al: Carbon-ion radiotherapy in osteosarcoma of the mandible: a case report. J Korean Assoc Oral Maxillofac Surg 2021

grade $^{7}$. Preoperative chemotherapy can be performed after reassessment of the lesion with various imaging modalities including MRI, PET-CT, and chest CT. If the tumor is considered resectable, wide excision can be done with adjuvant treatment of chemo- and radiotherapy. A literature review indicates that the current principle of treatment for osteosarcoma of the extremities is neo-adjuvant chemotherapy prior to surgery and subsequent adjuvant chemotherapy, but the principle of treatment for head and neck osteosarcoma involves a primarily multidisciplinary approach. The major component of multidisciplinary management is adequate surgical resection with wide margins ${ }^{8}$. The benefits of both neoadjuvant and adjuvant chemo/radiotherapy remain controversial. Osteosarcomas are known to be relatively resistant to conventional photon radiation therapy, which makes radia- 
tion therapy insignificant as a primary modality. On the other hand, the benefits of radiotherapy are the reduction of local recurrence and improvement of overall survival for patients with positive/uncertain surgical margins ${ }^{9,10}$.

CIRT is a heavy ion radiotherapy that uses a particle beam of carbon ions. Particle beam is different from conventional radiotherapy in both its physical and biological characteristics. In conventional radiotherapy, a photon releases energy continuously through biological objects, thus the dose is distributed roughly equally throughout the path. In particle beams, however, the energy is released at the inverse of the velocity $^{11}$. The particle beams are accelerated very fast (approximately $70 \%$ of the speed of light) when they enter the patient's body, thus the interaction between healthy tissue and the beam is low at the entry. However, when the speed drops as the beam pierces deeper into the patient's tissues, the beam deposits almost all its energy at a certain velocity, suddenly rising to a peak when the particles ultimately stop (the "Bragg peak").(Fig. 4) Furthermore, the dose deposition decreases rapidly after the Bragg peak, so the toxicity is significantly reduced by dose-sparing of normal and untargeted structures ${ }^{12}$. The charged particle beam can be adjusted to reach the Bragg peak precisely at the tumor. Other particle beams, such as protons, have a similar dose distribution as carbons. However, compared to protons, carbon with its larger mass decreases beam scattering, resulting in a sharper dose distribution border with minimal penumbra ${ }^{13}$. Therefore, CIRT is expected to suppress tumor growth and reduce complications of normal tissues, potentially more efficiently than protons or X-rays.

Linear energy transfer (LET) is defined as the amount of radiation energy released to the surroundings per unit length as it passes through the medium. Because of the large LET of the carbon beam, the carbon beam has a biological effect that is significantly different at the DNA level. Relative biological effectiveness (RBE), a measure of the biological potency of a particular radiation modality, is defined as the ratio of a reference dose (usually X-rays of $250 \mathrm{kVp}$ ) to the test radiation dose in causing the same tumor kill. Therefore, the RBE of a photon is 1 . Although RBE is a complex value affected by many variables (the LET of the test radiation, physical dose, type of tumor irradiated, depth of tumor, end point, etc.), generally speaking, the RBE for a proton is considered to be 1.1, and the RBE for a carbon ion is accepted to be 2-3 or higher ${ }^{14}$.

The dose distribution of CIRT is advantageous. However, it is not perfect because the range of the Bragg peak is uncertain at the distal end, the range is sensitive to set-up variation, anatomical change can occur inter-fractionally, and there can be tumor motion. Despite these obstacles, it is believed that CIRT can deliver higher energy to deep tumors than photonor proton-based treatments while simultaneously saving nearby radiosensitive structures ${ }^{15}$. Although no phase III randomized trial data exist, the reported oncologic outcomes and side effect profiles of CIRT are very encouraging ${ }^{16}$. Because the infrastructure is costly, transnational collaborations are necessary to provide the basis of benefit ${ }^{14}$.

Patients with sarcomas originating in the head and neck region have the lowest overall survival rates among those with sarcomas of other body parts ${ }^{17}$. Jingu et al. ${ }^{18}$ reported that the overall survival rate of patients with head and neck osteosarcoma treated with CIRT was superior to that in past studies using conventional therapy. The 3-year overall survival rate was $44.4 \%$, whereas the reported 3 -year survival rates for patients with unresectable osteosarcoma of the head and neck are $30 \%$ or less ${ }^{18}$. Compared with conventional radiotherapy
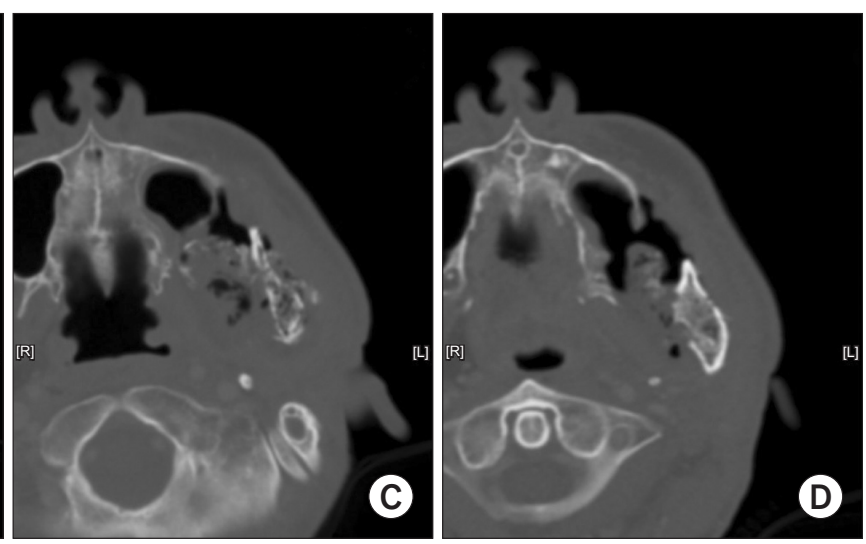

Fig. 5. A. October 2016. Osteolytic lesion on left mandibular condyle with pathologic fracture. B. March 2018. C. November 2018. Necrotic change was noted. D. January 2019.

Tae-Wook Ha et al: Carbon-ion radiotherapy in osteosarcoma of the mandible: a case report. J Korean Assoc Oral Maxillofac Surg 2021 
and CIRT with lower doses, CIRT with 70.4 GyE/16 fractions for bone and soft-tissue sarcoma of the adult head and neck is considered effective with acceptable toxicities ${ }^{18}$. No other studies of dose-escalation were found in our search of the literature.

In some case reports, CIRT showed a positive effect when combined with subsequent surgical removal of recurrent osteosarcoma. Kohyama et al. ${ }^{19}$ reported performing salvage and reconstructive surgery for the recurrence of osteosarcoma after CIRT. No postoperative complications, such as woundhealing delay, were observed, so early initiation of adjuvant chemotherapy was possible ${ }^{19}$. In our case, pulmonary metastases posed an obstacle to surgical resection of the primary lesion following CIRT. Our patient had necrotic bone falling out of the mandible 2 years after the CIRT since December 2018.(Fig. 5) Sasahara et al. ${ }^{20}$ reported that volumes receiving more than $50 \mathrm{GyE}$ and the presence of teeth within the planning target volume were risk factors for the development of osteoradionecrosis (ORN) after CIRT. Therefore, reduction of the bone volume exposed to a high radiation dose may prevent $\mathrm{ORN}^{20}$. In our case, the patient underwent palliative radiotherapy as well as CIRT, so the risk for ORN was higher.

Heavy ion therapy is still in its infancy compared with other radiotherapy modalities. However, technological advancements such as LET painting, mixed beam strategy which adopts oxygen ions for hypoxic tumors, and improvement of motion management technologies make the future of heavy ion therapy promising ${ }^{14}$. Thus, CIRT is likely to play a major role as a future radiotherapy modality.

\section{ORCID}

Tae-Wook Ha, https://orcid.org/0000-0002-8219-1204

Slmaro Park, https://orcid.org/0000-0002-2018-7509

Min Yeong Youn, https://orcid.org/0000-0003-2081-8953

Dong Wook Kim, https://orcid.org/0000-0001-6167-6475

Hyung Jun Kim, https://orcid.org/0000-0002-3364-9995

\section{Authors' Contributions}

T.W.H. prepared original draft of the manuscript. S.P. conceptualized this manuscript. M.Y.Y. prepared the patient data and prepared for the journal submission. D.W.K. revised and corrected the manuscript. H.J.K. participated in article design and he carefully reviewed and revised the manuscript for final approval. All authors read and approved the final manuscript.

\section{Ethics Approval and Consent to Participate}

Retrospective data collection was approved by the Institutional Review Board of Yonsei University Dental Hospital (IRB No. 2-2019-0018).

\section{Conflict of Interest}

No potential conflict of interest relevant to this article was reported.

\section{References}

1. Lim S, Lee S, Rha SY, Rho JK. Cranofacial osteosarcoma: single institutional experience in Korea. Asia Pac J Clin Oncol 2016;12:e149-53. https://doi.org/10.1111/ajco.12072

2. Sturgis EM, Potter BO. Sarcomas of the head and neck region. Curr Opin Oncol 2003;15:239-52. https://doi.org/10.1097/00001622200305000-00011

3. Schwarz R, Bruland O, Cassoni A, Schomberg P, Bielack S. The role of radiotherapy in oseosarcoma. In: Jaffe N, Bruland OS, Bielack S, eds. Pediatric and adolescent osteosarcoma. Cancer treatment and research. Boston: Springer; 2010:147-64.

4. Jeong HI, Lee MJ, Nam W, Cha IH, Kim HJ. Osteosarcoma of the jaws in Koreans: analysis of 26 cases. J Korean Assoc Oral Maxillofac Surg 2017;43:312-7. https://doi.org/10.5125/jkaoms.2017.43.5.312

5. Machak GN, Tkachev SI, Solovyev YN, Sinyukov PA, Ivanov SM, Kochergina NV, et al. Neoadjuvant chemotherapy and local radiotherapy for high-grade osteosarcoma of the extremities. Mayo Clin Proc 2003;78:147-55. https://doi.org/10.4065/78.2.147

6. Delaney G, Jacob S, Featherstone C, Barton M. The role of radiotherapy in cancer treatment: estimating optimal utilization from a review of evidence-based clinical guidelines. Cancer 2005;104:1129-37. https://doi.org/10.1002/cncr.21324

7. Neville B, Damm DD, Allen C, Chi A. Oral and maxillofacial pathology. 4th ed. Philadelphia: Saunders; 2015.

8. Krishnamurthy A, Palaniappan R. Osteosarcomas of the head and neck region: a case series with a review of literature. J Maxillofac Oral Surg 2018;17:38-43. https://doi.org/10.1007/s12663-017$1017-8$

9. Makary RF, Gopinath A, Markiewicz MR, Fernandes R. Margin analysis: sarcoma of the head and neck. Oral Maxillofac Surg Clin North Am 2017;29:355-66. https://doi.org/10.1016/ j.coms.2017.04.002

10. Kimura Y, Tomihara K, Tachinami H, Imaue S, Nakamori K, Fujiwara $\mathrm{K}$, et al. Conventional osteosarcoma of the mandible successfully treated with radical surgery and adjuvant chemotherapy after responding poorly to neoadjuvant chemotherapy: a case report. J Med Case Rep 2017;11:210. https://doi.org/10.1186/s13256-017-1386-0

11. Ebner DK, Kamada T. The emerging role of carbon-ion radiotherapy. Front Oncol 2016;6:140. https://doi.org/10.3389/ fonc. 2016.00140

12. Kong L, Gao J, Hu J, Lu R, Yang J, Qiu X, et al. Carbon ion radiotherapy boost in the treatment of glioblastoma: a randomized phase I/III clinical trial. Cancer Commun (Lond) 2019;39:5. https://doi. org/10.1186/s40880-019-0351-2

13. Durante M, Paganetti H. Nuclear physics in particle therapy: a review. Rep Prog Phys 2016;79:096702. https://doi. org/10.1088/0034-4885/79/9/096702

14. Mohamad O, Yamada S, Durante M. Clinical indications for carbon ion radiotherapy. Clin Oncol (R Coll Radiol) 2018;30:317-29. 
https://doi.org/10.1016/j.clon.2018.01.006

15. Blattmann C, Oertel S, Schulz-Ertner D, Rieken S, Haufe S, Ewerbeck V, et al. Non-randomized therapy trial to determine the safety and efficacy of heavy ion radiotherapy in patients with nonresectable osteosarcoma. BMC Cancer 2010;10:96. https://doi. org/10.1186/1471-2407-10-96

16. Mohamad O, Makishima H, Kamada T. Evolution of carbon ion radiotherapy at the National Institute of Radiological Sciences in Japan. Cancers (Basel) 2018;10:66. https://doi.org/10.3390/cancers10030066

17. Ballo MT, Zagars GK, Cormier JN, Hunt KK, Feig BW, Patel $\mathrm{SR}$, et al. Interval between surgery and radiotherapy: effect on local control of soft tissue sarcoma. Int J Radiat Oncol Biol Phys 2004;58:1461-7. https://doi.org/10.1016/j.ijrobp.2003.09.079

18. Jingu K, Tsujii H, Mizoe JE, Hasegawa A, Bessho H, Takagi R, et al.; Organizing Committee for the Working Group for Head-andNeck Cancer. Carbon ion radiation therapy improves the prognosis of unresectable adult bone and soft-tissue sarcoma of the head and neck. Int J Radiat Oncol Biol Phys 2012;82:2125-31. https://doi. org/10.1016/j.ijrobp.2010.08.043

19. Kohyama K, Yamada K, Sugiura H, Hyodo I, Ozawa T, Hasegawa $\mathrm{Y}$, et al. Salvage surgery and microsurgical reconstruction for recurrence of skull base osteosarcoma after carbon ion radiotherapy. Nagoya J Med Sci 2015;77:667-73.

20. Sasahara G, Koto M, Ikawa H, Hasegawa A, Takagi R, Okamoto Y, et al. Effects of the dose-volume relationship on and risk factors for maxillary osteoradionecrosis after carbon ion radiotherapy. Radiat Oncol 2014;9:92. https://doi.org/10.1186/1748-717X-9-92

How to cite this article: Ha TW, Park S, Youn MY, Kim DW, Kim

HJ. Carbon-ion radiotherapy in osteosarcoma of the mandible: a case report. J Korean Assoc Oral Maxillofac Surg 2021;47:315320. https://doi.org/10.5125/jkaoms.2021.47.4.315 